\title{
¿Que Moral Para un Derecho Intercultural? La Autonomía y el Concepto "SimpliciteR" DE TOLERANCIA ${ }^{1}$
}

\author{
Juan Pablo Zambrano Tiznado
}

RESUMEN: El objetivo del presente trabajo es doble. En primer lugar, pretendo defender un concepto simpliciter de tolerancia según el cual la autonomía personal o la necesidad de fortalecer una cultura son las únicas razones que debe ponderar el agente-Estado para tolerar. En segundo lugar, pretendo responder afirmativamente a la pregunta de si puede el liberalismo perfeccionista y multicultural de Joseph Raz sostener desde la teoría moral y política un derecho intercultural. Para cumplir estos objetivos, me concentraré en los usos morales del término tolerar para luego siguiendo a Michael Sandel, analizar los casos de tolerancia crítica y no crítica. Posteriormente, desarrollaré el liberalismo de Raz con el fin de mostrar los problemas teóricos que genera el fundamento de los derechos colectivos. Concluiré afirmando la existencia de derechos colectivos como recurso institucional para permitir el desarrollo de grupos culturales minoritarios dentro del actual desarrollo del Estado- nación.

PALABRAS CLAVE: Tolerancia. Autonomía personal. Cultura minoritaria. Liberalismo multicultural.

\section{INTRODUCCIÓN}

En publicaciones anteriores he mostrado como los tribunales chilenos han condenado a mapuches en delitos culturalmente motivados (Zambrano y Agüero 2009). Lo que me interesa destacar ahora es que independiente de las

\footnotetext{
1 Agradezco al Dr. Michel Duquesnoy por sus valiosos comentarios. Evidentemente, los posibles errores del texto son de mi exclusiva responsabilidad.

2 Escuela de Derecho, Universidad Arturo Prat, Chile. Becario Conicyt y Doctorando en Ciencias Humanas mención Discurso y Cultura, Universidad Austral, Chile. Correo electrónico: juanpablo. zambranotiznado@gmail.com
} 
razones jurídicas involucradas, los mapuches insisten en que el Estado chileno no es tolerante con su cultura y entonces, el reclamo sería que se utiliza al derecho como mecanismo de asimilación cultural. Se trata de que una cultura mayoritaria, la chilena, impone su sistema de valores a quienes tienen, en algunos aspectos, un sistema moral distinto. Este problema no es nuevo ni exclusivo de nuestra sociedad y es común escuchar en sede judicial a distintos grupos culturales que realizan este mismo tipo de alegaciones. Sin embargo, el problema no es jurídico sino político y más precisamente moral. El derecho, como fenómeno social, requiere un fundamento desde la ética y la filosofía política porque evidentemente sus disposiciones no son asépticas. En efecto, detrás de un sistema jurídico hay una determinada forma de comprender la vida en comunidad y una concepción de lo bueno y lo malo. El objetivo de este trabajo es a) argumentar a favor de un concepto focal de tolerancia que releve a la autonomía como única razón para tolerar y b) sostener que una particular concepción del liberalismo, el liberalismo perfeccionista y multicultural de Joseph Raz, permite fundamentar dentro del Estado-nación un derecho intercultural que permita a las culturas minoritarias ser titulares de ciertos derechos colectivos posibilitando con ello tanto su florecimiento como el desarrollo pleno de sus miembros. Ambas tesis tienen importantes consecuencias en nuestra comprensión del papel del Estado en sociedades multiculturales porque, si asumimos que las culturas minoritarias que habitan un mismo territorio actúan como unidades de agencia entonces me parece, podemos fundamentar la existencia de derechos y deberes colectivos. En otras palabras, sostendré que solo la tolerancia basada en la autonomía es tolerancia en sentido simpliciter y a partir de este argumento justificaré el deber del Estado de tolerar ciertos derechos colectivos tales como el derecho a la lengua y a la cultura en el caso de las culturas minoritarias cuando actúan como agencia. Ahora bien, en este trabajo asumo dos cuestiones. En primer lugar, que pese a los progresos del orden jurídico internacional y a la existencia de fenómenos como la globalización, los sistemas jurídicos siguen siendo nacionales. En segundo lugar que, más allá de nuestras divergencias, la teoría moral y política que sostiene la comprensión del "derecho" al menos en occidente es el liberalismo. En lo que sigue, procederé a concentrarme en los usos morales de la tolerancia para luego, a partir de la distinción de Michael Sandel entre tolerancia crítica y no crítica, defender un concepto simpliciter de tolerancia centrada en la autonomía. Luego defenderé que es posible fundamentar un derecho intercultural a partir del liberalismo perfeccionista y multicultural de Joseph Raz. 


\section{TOLERANCIA Y CONFLICTO MORAL}

La tolerancia implica siempre un conflicto moral. En el fondo, la pregunta básica del agente (en términos institucionales el Estado) es si, pese a la tendencia a prohibir una determinada práctica, existen mejores razones para permitirla. Esta pregunta propia del razonamiento práctico, implica que el Estado deberá ponderar razones y decidir. En nuestras sociedades multiculturales son los legisladores y los tribunales de justicia, cuando actúan discrecionalmente, quienes deben responder esta pregunta. Entonces, como ha mostrado Garzón Valdés (2000, p. 183), un elemento del concepto es la “[...] ponderación de los argumentos a favor de la permisión o prohibición del acto en cuestión". Si esto es así, un caso secundario de uso del término tolerancia sería la que Sandel (1996) llama "no crítica", cuyo ejemplo paradigmático corresponde a la tolerancia rawlsiana (SANDEL, 1996). Como se sabe, Rawls (1985), al vincular la tolerancia a un concepto de justicia como equidad (por tanto política y no metafísica), evita que el agente pueda contrastar la bondad moral de una práctica con una determinada doctrina omnicomprensiva en coherencia con su defensa de la neutralidad del Estado (RAWLS, 1993).

Un caso distinto está constituido por la "tolerancia crítica" (SANDEL, 1996). Esta tolerancia es entendida como admitiendo

[...] una evaluación sobre el valor moral o permisible de la práctica en discusión, y, en consecuencia, permitiendo o restringiendo dicha práctica de acuerdo al peso de las consideraciones morales en conflicto dependientes del juicio moral. (CORREA, 2006, p. 106).

Ejemplos de ella serían las conceptualizaciones de Tomás de Aquino (S. Th. II-II, a.10, p. 11), Ernesto Garzón Valdés (2000) y Joseph Raz (1986; 1999).

\section{TOLERANCIA CRÍtica}

La tolerancia crítica (SANDEL, 1996), admite diversas razones morales o prudenciales para justificar o no la permisión de una determinada práctica, de modo que este tipo de tolerancia admite la posibilidad de justificar casos de no tolerancia. En el caso de Tomás de Aquino (S. Th. II-II, a.10, p.11) la razón para tolerar el culto de los judíos era evitar mayores males o conseguir bienes mayores, para Garzón Valdés (2000, p. 182) se tolera “[...] en aras de un valor que el tolerante considera superior como el libre desarrollo de la 
personalidad o la buena relación con los hijos” en fin, para Raz (1999, p. 100), se tolera porque el principio de autonomía personal implica poder elegir entre diversas opciones virtuosas pero incompatibles. En los ejemplos, Tomas de Aquino podría afirmar "tolero el culto de los mapuches porque los perjuicios de no tolerar son mayores que los perjuicios de tolerar dicho culto". Garzón Valdés, podría afirmar "tolero que los amigos de mi hijo no me saluden, porque de no tolerar dicha práctica se afecta la buena relación que tengo con él" o, en un plano más institucional, "el Estado tolera que los mapuches resuelvan sus conflictos de acuerdo a su propio derecho porque de no tolerar dicha práctica se perjudicarían aun más las relaciones que el Estado tiene con ellos". En este caso, es el mayor valor que el Estado le asigna a las buenas relaciones con la cultura minoritaria la razón por la que se tolera. Ahora bien, Raz podría afirmar "el Estado tolera las relaciones sexuales consentidas entre mapuches independiente de la edad de la mujer ${ }^{3}$ porque quienes las realizan están ejerciendo su autonomía personal”. ${ }^{4}$ En los ejemplos, las referencias a las razones son un punto crucial y diferenciador. En los casos de Tomas de Aquino y de Garzón Valdéz, se instrumentaliza a las personas o culturas minoritarias porque las razones para tolerar son ajenas a ellos de modo que, "la tolerancia" es un medio para que el agente-Estado alcance otros fines. En Tomas de Aquino, ese único fin es evitar los perjuicios que se pueden derivar de no tolerar el culto de los judíos y en Garzón Valdés, se tolera para evitar los efectos negativos que pudiera tener en la relación con el Estado no aceptar la autoridad del Derecho

3 Para la cultura mapuche una mujer esta sexualmente disponible desde su primera menstruación independiente de su edad. En Chile, el art. 361 del Código Penal tipifica como violación impropia a quien acceda carnalmente a una persona menor de 14 años. Esta norma ha sido usada en Chile para condenar a mapuches que han tenido relaciones sexuales consentidas con menores de 14 ańos en la creencia de que actuaban conforme a una norma de su cultura que permite su conducta. Cf. ZAMBRANO; AGÜERO, 2009.

${ }^{4}$ Si la relación es consentida podría ser considera un bien porque fue una elección libre entre otras opciones valiosas pero incompatibles. Sin embargo, también podría ser un caso de un "mal inofensivo o sin víctimas" y pese a que en Raz (1999, p. 111; 1986, p. 418-419) la autonomía sólo sirve para fundamentar la tolerancia de bienes, este tipo de males se deben tolerar en razón del daño a la autonomía que sufriría el agente por el carácter global e indiscriminado de la coacción. En otras palabras, pese a ser un mal y por tanto no estar justificada la tolerancia, se debe tolerar porque la coacción que sufriría el agente es de tal entidad que no se justificaría. Cf. ZAMBRANO, 2011. Una tercera opción es considerar la posibilidad de que la acción constituya una acción moralmente mala en que existe una víctima. He argumentado que ello sería posible si entendemos como un mal la perdida de una oportunidad por parte de la menor, esto es, la perdida de la posibilidad de elegir la virginidad. En esta línea argumental lo importante es que por la edad en que se realizan estas acciones —antes de los 14 años- es posible cuestionar si la menor podía elegir libremente entre diferentes opciones y por tanto si al tener relaciones sexuales ella quedo peor de lo que hubiera estado si hubiese tenido la posibilidad de elegir. En este caso, el daño es la perdida de una chance. Cf. ZAMBRANO; AGÜERO, 2009. 
propio del pueblo Mapuche. A diferencia de dichos casos, en el ejemplo de Raz, la razón para tolerar implica reconocer que la persona o cultura minoritaria es un fin en sí mismo porque, es la valoración de la autonomía la que justifica el cambio del estatuto deóntico de la conducta que le desagrada. De este modo, toleramos las prácticas culturales de la cultura mapuche porque reconocemos que actúan como una unidad de agencia. Así también, toleramos las prácticas de los mapuches porque reconocemos que ellos tienen derecho a vivir de acuerdo a sus elecciones y entonces, de este modo pueden concretar, dentro de una cultura robusta, sus planes de vida felicitante. Mi tesis es que el sentido focal de la tolerancia debe precisar que es sólo la autonomía la razón que debe ser ponderada por el Estado tolerante. Pensemos en un Estado que decide tolerar la medicina mapuche en hospitales públicos pese a considerarla un mal. De hecho, imaginemos que no existen antecedentes científicos que muestren las bondades médicas de la medicina mapuche y agreguemos que la razón del Estado para cambiar el estatuto deóntico de la conducta es electoral. Así, los analistas electorales de este Estado, argumentan que los mapuches se sentirán acogidos y valorados por el Estado. Ahora bien, ¿este Estado "tolera" la medicina mapuche? Si respondemos afirmativamente ¿podemos decir que esta tolerancia es idéntica a la de otro Estado que tolera la misma práctica pero argumentando que las personas tienen derecho a cuidar su salud según ellas decidan mientras no atenten contra el principio de dańo? La respuesta es intuitiva, pero no por ello menos fuerte. En ambos casos, la acción que se tolera es considerada un mal moral (el "aparente" mal moral consistiría en promover como medicinal prácticas que científicamente no tienen ese carácter) pero la diferencia estriba en que admitir razones electorales $u$ otras similares implica usar a las culturas minoritarias como medios para conseguir otros propósitos quitándoles su carácter de fin en sí mismas. Admitir a la autonomía como la única razón que justifique tolerar es reconocer en los demás la posibilidad que tienen de hacerse cargo de su propia vida.

\section{El liberalismo multicultural de RaZ Y LOS DERECHOS COLECTIVOS}

Asumir el papel del liberalismo como teoría política y moral (RAZ, 2001, p. 190) que sostiene ideológicamente la concepción del derecho vigente en occidente implica reconocer que nuestros sistemas jurídicos se caracterizan tanto por la defensa de un estado neutral como por la defensa de derechos individuales (CRISTI, 1998, p. 48). Entonces, la idea de un liberalismo perfeccionista y multicultural parece contradictoria. Sin embargo, 
Raz modifica la teoría liberal tanto al redefinir el rol de la cultura (2001), de modo que pueda sostener la existencia de derechos colectivos, como al afirmar el carácter perfeccionista del liberalismo (1999) al relevar a la autonomía personal como el principal valor que esta teoría defiende.

Raz (2001, p. 192) argumenta que la persona solo puede desarrollarse en plenitud dentro de una cultura floreciente de modo que el liberalismo debe propender al desarrollo de cada cultura porque con ello creara las condiciones de posibilidad para que las personas construyan y concreten los planes de vida que autónomamente han elegido. Entonces, valorar la autonomía personal implica sostener que las culturas minoritarias tienen derecho a ejercer las prácticas culturales que estimen convenientes.

El problema del liberalismo clásico es que la agencia es siempre individual sin dar lugar a la existencia de derechos colectivos (GRIFFIN, 2003 , p. 160ss). En otras palabras, la agencia individual puede dar lugar a derechos y deberes que benefician al grupo pero en todo caso los derechos y deberes seguirán siendo individuales. En contra, Raz (1986, p. 208; GREEN, 2005, p. 516ss) argumenta que en el caso del deber de tolerancia y su correlato el derecho a ser tolerado habría un bien colectivo que justifica derechos y deberes por el bien del grupo.

En el caso de los mapuches, se ha argumentado en sede penal que tienen la costumbre de considerar a las mujeres como sexualmente disponibles desde la primera menstruación independiente de su edad. En cambio, para el derecho chileno, existe delito de violación en caso de una relación sexual con una persona menor de 14 años aunque exista consentimiento. Según Raz, la cultura mayoritaria podría tener el deber de tolerar esta práctica cultural y la cultura minoritaria el derecho a ser tolerada. Sin embargo, queda pendiente la justificación teórica de los derechos colectivos (ZAMBRANO, 2009). El problema es mayor si consideramos que según Raz un mapuche solo podría desarrollarse plenamente si su cultura se hace más robusta de modo que los sistemas jurídicos deberían fortalecer y posibilitar el ejercicio de prácticas culturales imponiendo deberes colectivos para posibilitar el pleno desarrollo de todos los miembros de la cultura minoritaria. Para Raz (2001, p. 57ss.), alguien tiene derecho sólo si un aspecto de su bienestar es una razón para la existencia del deber. En esta conceptualización Raz está pensando en derechos individuales. Pero si asumimos que una cultura minoritaria puede actuar como una unidad de agencia que se beneficia del respeto por su lengua y prácticas culturales por parte de las demás culturas con las que comparte un territorio, 
entonces podemos fundamentar, en razón del bienestar de volverse más robusta que obtiene la cultura minoritaria, determinados deberes y derechos colectivos. Ya no se trata de un bienestar individual, se trata del bienestar que la cultura obtiene al volverse más robusta. Evidentemente, el bien comunal de poder desarrollar sin interferencias una actividad implica que un miembro $\mathrm{X}$ de la cultura minoritaria Y podrá desarrollar libremente una determinada práctica cultural pero, al defender la titularidad de determinados derechos colectivos de las culturas minoritarias, garantizamos su desarrollo, única forma que permitirá a sus miembros ejercer efectivamente su autonomía personal y desarrollarse plenamente.

Ahora bien, podría sostenerse que la cultura mayoritaria y el Estado tendrían entonces el deber de tolerar prácticas culturales que la cultura mayoritaria considera aberrantes como la oblación del clítoris en aras de respetar el derecho de la cultura minoritaria a ser tolerada. Pero ello no es necesariamente así. Me parece que la solución a este dilema pasa en primer lugar por perfeccionar el papel del principio de daño como elemento fundante de la tolerancia basada en la autonomía y por defender la posibilidad de los miembros de una cultura a salirse libremente de ella.

\section{Conclusión}

A partir de las reflexiones anteriores es posible afirmar que existen buenas razones para entender a la autonomía personal y a la necesidad de fortalecer una cultura minoritaria como las únicas razones que tiene el Estado para modificar el estatuto deóntico (de prohibido a permitido) de una práctica que considera un mal moral. Esta forma de entender a la tolerancia implica relevar el papel de las culturas como sujetos susceptibles de deberes y derechos, entre ellos el derecho a que se toleren sus prácticas culturales. Sin embargo, ni la propia cultura, ni la cultura mayoritaria pueden obligar a un agente moral a que realice o participe de prácticas culturales con la que no está de acuerdo sin violar su propia autonomía. Se trata entonces, de concebir los sistemas jurídicos nacionales como plenamente interculturales de modo que respeten tanto la autonomía personal como las prácticas culturales diversas aunque ellas atenten contra los valores de la cultura mayoritaria. De otro modo, si no se fortalecen las culturas minoritarias, sus miembros no podrán realizarse plenamente. Finalmente, me parece que a partir de nociones como las de agencia, de bienestar y de cultura es posible desarrollar una teoría de 
los derechos colectivos plenamente liberal que sea respetuosa no solo de las personas sino también de las culturas.

TIZNADO, Juan Pablo Zambrano. Autonomy and the concept of tolerance simpliciter: Which morals for intercultural rights? Trans/Form/Ação, Marília, v. 35, n. 3, p. 221-230, Set./Dez., 2012.

\begin{abstract}
This article has two objectives. First, I want to represent a concept of tolerance simpliciter where personal autonomy and the need to strengthen a culture are the only reasons for which the agent-state must consider tolerance. Secondly, I intend to respond affirmatively to the question of whether, in the perfectionist and multicultural liberalism of Joseph Raz, intercultural rights can be derived from moral and political theory. To meet these objectives, I will focus on the moral uses of the word "tolerate" and then, following Sandel, on the analysis of critical and uncritical tolerance. Subsequently, I will explore Raz' liberalism in order to show the theoretical problems generated by the foundations of collective rights. I conclude by affirming the existence of collective rights as an institutional resource for enabling the development of cultural minority groups within the current development of the nation state.
\end{abstract}

KEY WORDS: Tolerance. Personal autonomy. Minority culture. Multicultural liberalism.

\title{
REFERÊNCIAS
}

CORREA, Mauricio. Dos versiones rivales sobre la tolerancia. La crítica de Michael Sandel a John Rawls. Veritas, Casablanca, v. 1, n. 14, p. 97-119, 2006.

CRISTI, Renato. La critica comunitaria a la moral liberal. Estudios Públicos, Santiago, n. 69, p. 47-68, 1998.

GARZÓN VALDÉS, Ernesto. No pongas tus sucias manos sobre Mozart. Algunas consideraciones sobre el concepto de tolerancia. Instituciones Suicidas. Ciudad de México: Paídos Mexicana, 2000, p.181-198.

GREEN, Leslie. Three themes from Raz. Oxford Journal of Legal Studies, Oxford, v. 25, n. 3, p. 503-525, 2005.

GRIFFIN, James. Group right. In: MEYER, L; PAULSON, S.; POGGE, P. (Ed.). Right, culture and the law. Themes from the legal and political philosophy of Joseph Raz. Oxford: Oxford University Press, 2004, p. 161-182.

RAWLS, John. Justice as fairness: political not metaphysical. Philosophy and public affairs, Princeton, v.14, n. 3, p. 223-251, 1985.

. Political Liberalism. Nueva York: Columbia University Press, 1993. 
RAZ, Joseph. The morality of freedom. Oxford: Oxford University Press, 1986.

. Autonomía, tolerancia y el principio del daño. En: CORREA, R. (Trad.). Revista de Estudios Públicos, Santiago, n. 76, p. 91-113, 1999.

2001.

La ética en el ámbito público. En: MELON, L.M. (Trad.). Barcelona: Gedisa,

SANDEL, Michael. Judgemental toleration. In: GEORGE, R.P. (Edit.) Natural Law, Liberalism and Morality. New York: Oxford University Press, 2002, p. 107-112.

TOMÁS DE AQUINO. Suma Teológica. Madrid: Biblioteca de Autores Cristianos, 1990.

ZAMBRANO, Juan Pablo. Tolerancia, autonomía e inmoralidades “inofensivas" o "sin víctimas”. Critica, Revista Hispanoamericana de Filosofia, Ciudad de México, v. 43 n. 128, p. 81-86, 2011.

- Multiculturalidad y derechos colectivos en Joseph Raz. Isegoría, Revista de Filosofía Moraly Politica, Madrid, v. 41, p. 287-292, 2009.

; AGÜERO, Claudio. Multiculturalidad y discrecionalidad judicial en una sentencia penal: análisis desde Joseph Raz. Revista de Derecho de la Pontificia Universidad Católica de Valparaíso, Valparaíso, v. XXXII, p. 327-343, 2009.

Recebido em: 11.04.2012

Aceito em: 07.05.2012 
TIZNADO, J. P. Z. 